Please cite this article in press as: Eke et al., International data governance for neuroscience, Neuron (2021), https://doi.org/10.1016/

\title{
International data governance for neuroscience
}

\author{
Damian O. Eke, ${ }^{1,2}$ Amy Bernard, ${ }^{3}$ Jan G. Bjaalie, ${ }^{4}$ Ricardo Chavarriaga, ${ }^{5}$ Takashi Hanakawa, ${ }^{6}$ Anthony J. Hannan, ${ }^{7}$ \\ Sean L. Hill, ${ }^{8}$ Maryann E. Martone, ${ }^{9}$ Agnes McMahon, ${ }^{3}$ Oliver Ruebel, ${ }^{10}$ Sharon Crook, ${ }^{11}$ Edda Thiels, ${ }^{12}$ \\ and Franco Pestilli ${ }^{13, *}$ \\ ${ }^{1}$ Centre for Computing and Social Responsibility, De Montfort University, Leicester, UK \\ ${ }^{2}$ Human Brain Project \\ ${ }^{3}$ The Kavli Foundation, Los Angeles, CA, USA \\ 4 University of Oslo, Oslo, Norway \\ ${ }^{5}$ Center for Artificial Intelligence, School of Engineering, Zurich University of Applied Sciences, Zurich, Switzerland \\ ${ }^{6}$ Kyoto University Graduate School of Medicine, Kyoto, Japan \\ 7Florey Institute of Neuroscience and Mental Health, University of Melbourne, Melbourne, Australia \\ ${ }^{8} \mathrm{Krembil}$ Centre for Neuroinformatics, Centre for Addiction and Mental Health, Toronto, ON, Canada \\ 9University of California, San Diego, San Diego, CA, USA \\ ${ }^{10}$ Scientific Data Division, Lawrence Berkeley National Laboratory, Berkeley, CA, USA \\ ${ }^{11}$ School of Mathematical and Statistical Sciences, Arizona State University, Tempe, AZ, USA \\ ${ }^{12}$ National Science Foundation, Alexandria, VA, USA \\ ${ }^{13}$ Department of Psychology, Center for Perceptual Systems, Center for Theoretical and Computational Neuroscience, and Institute for \\ Neuroscience, University of Texas, Austin, TX, USA \\ ${ }^{*}$ Correspondence: pestilli@utexas.edu \\ https://doi.org/10.1016/j.neuron.2021.11.017
}

\section{SUMMARY}

As neuroscience projects increase in scale and cross international borders, different ethical principles, national and international laws, regulations, and policies for data sharing must be considered. These concerns are part of what is collectively called data governance. Whereas neuroscience data transcend borders, data governance is typically constrained within geopolitical boundaries. An international data governance framework and accompanying infrastructure can assist investigators, institutions, data repositories, and funders with navigating disparate policies. Here, we propose principles and operational considerations for how data governance in neuroscience can be navigated at an international scale and highlight gaps, challenges, and opportunities in a global brain data ecosystem. We consider how to approach data governance in a way that balances data protection requirements and the need for open science, so as to promote international collaboration through federated constructs such as the International Brain Initiative (IBI).

\section{INTRODUCTION}

The growing availability of shared neuroscience data from largeand small-scale projects is driving unprecedented research and innovation. As a result of a welcome move toward open sharing of neuroscience data, data are often crossing the legal and national borders from where they originate. The future of understanding the brain depends on developing a robust research ecosystem that facilitates bringing together data across diverse organismal sources, including human and non-human animals, collected under different jurisdictions. As a result of the international nature of many projects, neuroscience is creating novel opportunities for data sharing and discovery while also generating new technical, legal, and ethical challenges. These novel challenges depend, in part, on different laws and regulations across nations, states, institutions, and funders alike. As of today, the lack of global data governance coordination across countries often places the responsibility associated with data sharing on individual researchers and their institutions, increasing researchers' risk and liability or limiting the potential for discovery (Rabesandratana, 2019). Institutions that fear liability may err on the side of caution and interpret general regulations in a way that impedes sharing of scientific data (Box 1). There is a critical need to define and clarify neuroscience data governance across international borders. To facilitate scientific discovery, mitigating risks and data use safety concerns while minimizing liability to individual researchers should be made a top priority by researchers, institutions, professional societies, policy makers, industry, funders, and other stakeholders.

Data governance has been defined as the "overall management of the availability, usability, integrity, quality, and security of data in order to ensure that the potential of the data is maximized while regulatory and ethical compliance is achieved within a specific organizational context" (Fothergill et al., 2019). Historically, data sharing has been defined in a project-centric fashion, and in general, projects have been organized and managed within a single country or region. Importantly, we emphasize that data management is different from data governance, although the two are highly interconnected. Here, we define data governance as the principles, procedures, frameworks, 
Please cite this article in press as: Eke et al., International data governance for neuroscience, Neuron (2021), https://doi.org/10.1016/ j.neuron.2021.11.017

\section{CellPress} OPEN ACCESS

Box 1. Data protection regulatory challenges for international collaborations

The lack of clarity surrounding the EU's GDPR requirements and their varied interpretations have disrupted international data sharing collaborations. In 2019, Finland's National Institute for Health and Welfare stopped all data sharing with the laboratory run by US NIH director Dr. Francis Collins on account of GDPR-related concerns. This action disrupted decades of collaborative work on a project studying type 2 diabetes, which previously used 32,000 shared DNA samples. Similarly, the GDPR has been cited as the reason behind the International Genomics of Alzheimer's Project's restricting the sharing of data between partners outside the EU. The consortium now runs isolated analyses, which ultimately reduce the value of data, limit research, and cost additional money and time (Eiss, 2020; Rabesandratana, 2019). In another report (All European Academies et al., 2021), it is estimated that in 2019 , about 5,000 collaborative projects involving the NIH were affected by the implementation of the EU's GDPR.

and policies that ensure acceptable and responsible processing of data at each stage of the data life cycle, from collection, storage, processing, curation, sharing, and use to deletion. These procedures help maintain data integrity, quality, availability, accessibility, usability, and security and define data controllership (or stewardship) and other responsibilities related to the data. Data governance is rooted in existing laws, regulations, and ethical principles but extends beyond to include policies and interpretations within organizations and specific projects (Stahl et al., 2018). International data governance (IDG) therefore encompasses both standards and practices for ensuring that data are FAIR (findable, accessible, interoperable, and reusable) (Wilkinson et al., 2016) and the ethical principles, policies, recommendations, laws, and regulations that apply to processing, sharing, and using data within and across borders.

In this paper, we lay out the case for why a robust IDG is necessary for neuroscience and make recommendations on what parameters IDG should cover. Effective IDG should be compatible with the open-sharing needs of the neuroscience research community while respecting the diversity of ethics, cultures, and privacy around data sharing across nations. Critically, IDG should prioritize the ability of researchers around the world to work collaboratively and share data to better understand the brain. A key goal of IDG is to maximize sharing and impact generated from data and minimize the risk that researchers and institutions assume when sharing data. To achieve this goal, a key task of IDG is to clarify international policies and to help implement governance plans that facilitate research and respect the individuals (both investigators and study participants). As of today, the foundations of IDG are not established, and as a result, best practices for implementing IDG are also not agreed upon. Because of this, the discussion presented hereafter can benefit not only neuroscience research but also other fields in biomedical, behavioral, cognitive, and biological sciences. Likewise, neuroscience would benefit from any ongoing discussion in other scientific domains.

This paper arose from the Data Sharing and Standards Working Group established by the International Brain Initiative (IBI; https:// www.internationalbraininitiative.org; International Brain Initiative, 2020), a collective created to unify emerging national and regional large-scale neuroscience endeavors. The goal of this paper is to raise awareness of the need for clearer data governance frameworks for the neuroscience community and its stakeholders, including researchers, institutions, professional societies, publishers, funders, and policy makers, and to propose recommendations on how IDG can be established and managed.

\section{THE IMPORTANCE OF INTERNATIONAL DATA SHARING IN NEUROSCIENCE}

In recent years, neuroscience data sharing has finally been made a priority in the community (Ascoli et al., 2017; Avesani et al., 2019; Ferguson et al., 2014; McDougal et al., 2016; Milham et al., 2018; Nichols et al., 2017; Poldrack and Gorgolewski, 2014; Poline et al., 2012; Teeters et al., 2015). There are two primary drivers for this need. First, there is a critical need to increase the size of the datasets available, beyond what can be collected in individual laboratories, and to address the needs of emerging fields of research involving large neuroscience datasets (e.g., artificial intelligence [AI]; Kietzmann et al., 2019; Marblestone et al., 2016). Second, there has been a communitydriven need to address reproducibility (McDougal et al., 2016), openness, and FAIR-ness (Wilkinson et al., 2016). The movement for data sharing and openness has been strong, consistent, and successful (Klapwijk et al., 2021; Milham et al., 2018). As a result, the requirements and expectations for data management have moved from reluctance to open sharing and publication of datasets (Gorgolewski et al., 2016; Mackenzie, 2019).

Mechanisms for promoting the sharing of data have been or are currently being developed. As successful community standards and schemas such as the Brain Imaging Data Structure (BIDS; Gorgolewski et al., 2016; https://bids.neuroimaging.io), the Open Metadata Initiative for Neuroscience Data Structures (OpenMINDS; https://github.com/HumanBrainProject/openMINDS), and Neurodata Without Borders (NWB; https://www.nwb.org) emerge and gain traction, these efforts facilitate the distribution and reuse of data. The International Neuroinformatics Coordinating Facility (INCF; https://www.incf.org) has played a key role in the development and harmonization of technical standards for the international neuroscience community (Abrams et al., 2021). In turn, the Institute of Electrical and Electronics Engineers (IEEE) has made efforts to identify standardization priorities for neurotechnologies (Institute of Electrical and Electronics Engineers Standards Association, 2020). A distributed set of well-managed data archives has been established that can house neuroscience data of multiple types (e.g., brainlife. io, EBRAINS, Canadian Open Neuroscience Platform, dandiarchive.org, OpenNeuro.org, the National Institute of Mental Health [NIMH] Data Archive [NDA; https://nda.nih.gov], and NeuroMorpho.org). Funders are requiring or recommending posting data products on these archives. See Table S1 for IBI partner initiatives' data sharing policies and what they cover (Jwa and Poldrack, 2021). 
Please cite this article in press as: Eke et al., International data governance for neuroscience, Neuron (2021), https://doi.org/10.1016/ j.neuron.2021.11.017

\section{Neuron}

Box 2. Challenges to sharing spinal cord data across national borders

Neuroscience is characterized by multiple data archives, usually run by researchers, that cover specific data types or serve particular communities. Many of these repositories are recommended by journals and funders as a place to publish data and are therefore likely to serve an international clientele. The ODC-SCl (odc-sci.org) is a community platform hosted in the United States at the University of California, San Francisco, for sharing data in spinal cord injury. The majority of data are derived from translational research, but some de-identified human data are also hosted. Recently, a non-US researcher submitted de-identified human data to ODC-SCI. The curators were uncertain whether the data were de-identified according to HIPAA, as according to the data submission policy, data must be HIPAA compliant. The burden is on researchers to comply, which means that they will have to become familiar with standards outside of their own countries or regions. As indicated in Table 1 and Figure S1, concepts such as de-identification and anonymization may not have the same meaning across jurisdictions. Challenges as described in this use case will be encountered more frequently as data sharing through recognized repositories becomes more mainstream.

Although neuroscience has made great strides in establishing the basic infrastructure for data sharing and integration, these efforts focus mostly on technical standards. Still missing is a comprehensive IDG framework to guide a global neuroscience data ecosystem (i.e., the raw measurements, the metadata, and the software for analysis and management). As archives for neuroscience data are distributed globally, new mandates from both funders and publishers for data sharing to use these archives will increase the flow of data across international borders into data archives and back out again to the worldwide community. Thus, neuroscience is in the process of transforming from primarily a local, geographically limited, and lab-centric endeavor to an international data-centric activity whereby neuroscience data within these infrastructures may come from disparate sources, sites, or projects subject to different national and regional regulations, socio-cultural principles, and theoretical perspectives (Kellmeyer, 2018; Paninski and Cunningham, 2018; Teeters et al., 2015) (see Box 2). Furthermore, neuroscience research transcends not-for-profit scholarship to applied clinical or product-based outcomes (e.g., medical devices and consumer brain technologies; Statt, 2017; Urban, 2017; Wexler and Reiner, 2019; Vayena et al., 2016).

\section{THE EVOLVING DEFINITION OF NEUROSCIENCE DATA}

Here, we consider the question of whether IDG for neuroscience is covered under the broader issue of data governance for biomedical data (and data from other biological sciences) or whether there are unique aspects of the sharing of neuroscience data that require special consideration. Neuroscience is perhaps distinguished from other domains by its highly multidisciplinary nature, bringing together researchers with diverse expertise, including physiology, molecular biology, anatomy, medicine, behavior, cognitive science, and computational science. Each of these disciplines is served by its own research community with its own standards and model systems, which leads to a significant number of silos to cross when attempting to build infrastructures or forge collaborations. Moreover, neuroscience is, at the same time, also deeply integrative and brings together multiple disciplines, scales of biological organization, and data modalities to gain an in-depth picture of the nature and functions of the brain and other neural systems. Neuroscience data are characterized by constant technological flux as funders and scientists seek to develop new techniques that will push the traversal of scales and modalities, leading to new data types and infra- structures constantly being required. Neuroscience data can be gigantic. For instance, typical neuroimaging protocols produce nearly 50 GB of data per participant at a single visit, approaching a petabyte-order dataset from a large longitudinal cohort. Coordinating standards and infrastructures in the face of evolving technologies becomes especially challenging both within and across national boundaries. Although other fields certainly must navigate cross-disciplinarity, neuroscience is uniquely reliant on integrating and compiling heterogeneity to both characterize the complexities of the brain and keep pace with the rapid rate of discovery.

Neuroscience has also yet to agree on what data should be shared. Historically, neuroscience data have been defined as raw measurements of nervous system structure, operational properties, and function (Figure 1A). A modern definition of neuroscience data transcends raw measurements to comprise derived data as well as metadata that describe the full set of processing steps and analyses used to produce derived data (Amunts et al., 2019; Avesani et al., 2019; Halchenko and Hanke, 2012). These measurements can be collected with a wide variety of techniques spanning all the way from genetic, molecular, and cellular approaches to imaging, physiological, and electrophysiological approaches, laboratory analysis, audio and visual recordings, and behavioral observations. Yet most raw data are unfit for research; they require curation and preprocessing. These derived data can be essential for gaining understanding of the brain compared with raw data, yet derived datasets cannot unequivocally be separated from the complex series of processing steps used to generate them (Figure 1B). Fortunately, the ever-expanding collaboration among neuroscience, engineering, and computer science has created opportunities to track and capture derived data and processing steps so as to support an expansion of the notion of data in neuroscience.

\section{THE GROWING NEEDS AND CHALLENGES FOR SHARING NEUROSCIENCE DATA}

The nature of neuroscience data creates challenges for sharing that are not only technical in nature but also economic, ethical, and legal. Similar to human genomic data, heightened sensitivity with neuroscience data comes, in part, from the connection it has to human identity, identification, and personhood. Neuroscience research and innovation have been noted to provide "unprecedented possibilities for accessing, collecting, sharing, and manipulating information from the human brain" (lenca 
A NEUROSCIENCE MEASUREMENTS

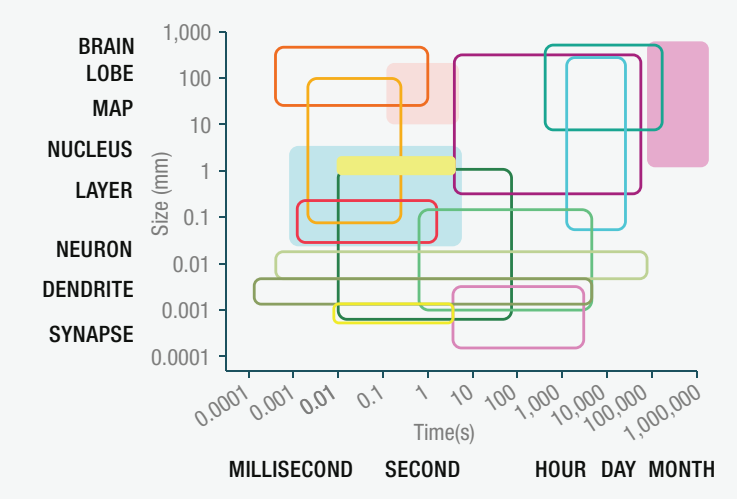

B NEUROSCIENCE DATA

EEG and MEG $\bigcirc$ vSD Imaging $\quad$ TMS $\bigcirc$ 2-DG Imaging $\bigcirc$ PET Imaging $\bigcirc$ fMRI Imaging $\bigcirc$ Brain Lesions $\bigcirc$ 0ptogenetics
Field Potentials $\bigcirc$ optogenetics $\bigcirc$ Light Microscopy $\bigcirc$ single Units $\bigcirc$ Patch Clamp $\bigcirc$ Calcium Imaging $\bigcirc$ Electron Microscopy $\bigcirc$ Microstimulation

Figure 1. Neuroscience is accompanied by the challenge of managing measurements and derived data across scales (A) Neuroscience measurements. Measurements in neuroscience have grown over the years. New image modalities and dimensions of measurement have contributed to understanding the brain.

(B) Neuroscience data. A modern definition of data in neuroscience is not limited to measurements but also encompasses derived data and analysis software, with all the associated metadata necessary to track the operations performed on the measurements to make them suitable for scientific projects.

and Andorno, 2017) in a way that uniquely challenges human rights principles. Possibilities of neuroprediction through neuroimaging studies (Haynes et al., 2007; Schreiber et al., 2013), neuromarketing (McClure et al., 2004), and pervasive neurotechnologies have informed considerations of NeuroRights (https:// neurorightsfoundation.org), a set of rights being proposed to be added to the Universal Declaration of Human Rights (UDHR) (lenca and Andorno, 2017; United Nations, 2021; Yuste et al., 2017). These issues highlight deeper concerns related to the continued convergence of neuroscience research and $\mathrm{Al}$ that may affect our understanding of human identity, free will, and privacy.

As in other fields, effective access to and use of neuroscience data creates tension between two fundamental community needs: maximizing data access and reducing risk to the subjects and researchers (Figure 2). On one hand, there is a need to increase openness, sharing, and reuse of data to advance scientific understanding and discovery (Ascoli et al., 2017; Avesani et al., 2019; Eglen et al., 2017; Ferguson et al., 2014; Milham et al., 2018; Nichols et al., 2017; Poldrack and Gorgolewski, 2014). On the other hand, there is a need to safeguard study participants, reduce risks associated with sharing identifiable information, and limit potential breaches in privacy (White et al., 2020). Additionally, the increasing use and sharing of neuroscience data in industry raise significant tensions related to commercialization and benefit sharing.

Although issues regarding ethics and subject protections are usually thought of in the context of human research, animal research also presents challenges, as public attitudes, rules, and ethical guidelines for experimenting on animals differ across countries (see Box 3). However, unlike human neuroscience data, which are increasingly regulated in many countries and re- gions, animal data are not yet regulated, raising possibilities of ignoring potentially problematic sharing of animal data (especially when the data are generated from countries with weak animal welfare regulations). Globally accepted governance mechanisms for animal data via IDG can therefore help harmonize procedures and processes for animal experimentations to meet scientific standards and also societal expectations.

In principle, neuroscience could benefit from IDG instruments and frameworks developed in other domains of biological sciences. In practice, after reviewing a wide range of subfields within the biomedical sciences, no established IDG framework was found. A set of data-related tools for IDG has been proposed in genomics research. The Global Alliance for Genomics and Health (GA4GH) has developed useful data tools such as Data Use Ontology version 1 (https://github.com/EBISPOT/DUO) and GA4GH Passport version 1 (https://github.com/ga4ghduri/ga4gh-duri.github.io/blob/master/researcher_ids/

ga4gh_passport_v1.md\#ga4gh-passport), which address the specific needs of genomic data. Although neuroscience could benefit from these tools, neuroscience data are likely to present additional technical and ethical challenges because of their complexity and scale. In light of recent restrictive regulations spearheaded by the European Union (EU) and Australian government, and with the growing need to share data across laboratories in different countries, an increasing legal burden is affecting both investigators and institutions. At the same time, improved ethical scrutiny for the legitimate use and reuse of neurodata shared across countries is necessary but lacks foundations (Hallinan et al., 2021). Neuroscience presents an excellent example for governments, international organizations, and other agencies to consider when developing and implementing data sharing policies. In the following sections, we outline challenges 


\section{Neuron} Review

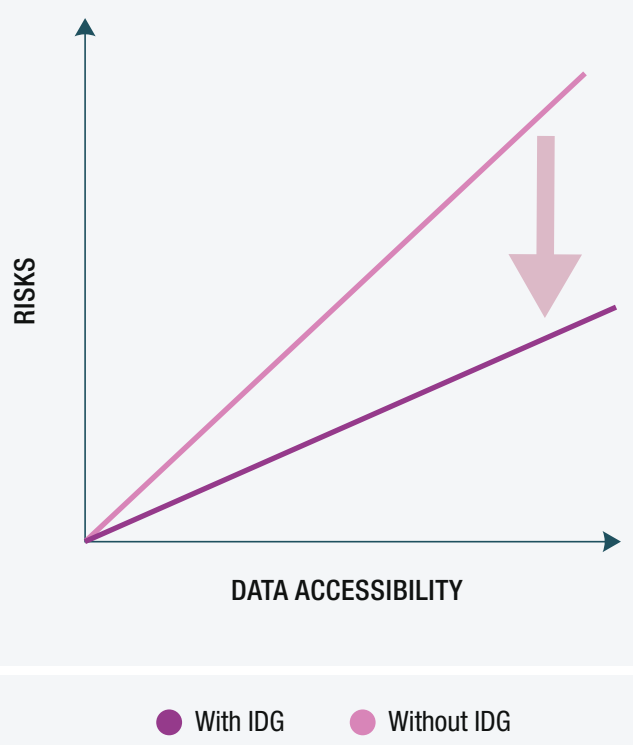

Figure 2. Data accessibility versus risks associated with sharing This figure shows the reduction in risks (arrow) associated with data sharing and accessibility as a result of proper international data governance (IDG). Without IDG, the risks increase at a higher rate than with IDG (pink). Clarity and facilitation of understanding of regulations helps mitigate risks to individual researchers and institutions.

in international data sharing and make recommendations in the context of neuroscience, recognizing that these issues may hold for other domains as well.

We touched on some of the broad technical, legal, and ethical challenges above, but there are also practical difficulties imposed by differences in language, cultural practices, and the multiplicity of technical standards. For example, during the course of this work, we gathered the data sharing policies governing the large brain projects or initiatives that are members of the IBI (Table S2; Organisation for Economic Co-operation and Development, 2017a). These documents were produced for national constituencies and so were not always written in a language understandable to those outside of these jurisdictions. Table 1 summarizes some of the types of challenges encountered in neuroscience data sharing that can be addressed by an IDG framework. In the following, we consider ethical and legal challenges in more detail.

Underlying a responsible IDG framework are binding regulations and ethical principles. Although many of the legal issues are addressed by the disparate data protection laws available in different regions and countries, some of the ethical issues, such as privacy and confidentiality, informed consent, and autonomy, have long histories in bioethics and technology ethics. Thus, their consideration often relies on soft-law governance instruments (see below). In the face of novel uses of globally available large and complex biomedical data occasioned by advancements in technology, a growing body of literature is emerging on the considerations of ethics of data in biomedical sciences (lenca et al., 2018; Knoppers and Thorogood, 2017;
Mittelstadt and Floridi, 2016; Salerno et al., 2017; GA4GH.org). This attention to ethics is important not only because of the expanding nature and role of data but also because of the potential risks of advancing research in direction breaching ethical principles not covered in law or the potential risk of lack of progress stemming from fear of breaching unclear ethical and legal principles.

A recent publication by the IBI Neuroethics Working Group discusses extensively many of the ethical issues associated with neuroscience data (such as informed consent, agency and autonomy, privacy, equitable access and benefits, misuse, dual use, and animal protection) and how different social norms, cultural practices, and religious values influence the way they are defined, operationalized, and enforced (Rommelfanger et al., 2018). These differing perspectives on the nature and meaning of ethics give rise to tensions in the context of global data sharing, especially in the presence of competing values, interests, and commitments. For most researchers, interactions with ethics compliance remain within local, regional, institutional, or project-specific processes that are different in jurisdictions in which their data are shared and received. Fothergill et al. (2019) also pointed out that the apparent dominance of European and North American perspectives in the field of neuroscience creates an imbalance in a research ecosystem that is increasingly global. Thus, the challenge for an IDG framework in neuroscience is to harmonize these different cultural and ethical perspectives in a way that will enhance understanding, advance collaborations, and facilitate responsible sharing of data. Leaving ethics behind in the global governance of neuroscience data may likely result in missed opportunities for collaboration and sharing, including benefit sharing, a concept emphasized by the Nagoya Protocol on Access and Benefit-sharing (Secretariat of the Convention on Biological Diversity, 2011).

\section{DATA-RELATED GOVERNANCE INSTRUMENTS}

As indicated above, international data sharing is typically subject to a set of norms comprising national and international legislation, recommendations, policies, and agreements in need of harmonization. These regulations are jurisdictionally constrained and fragmented but can have international implications (see Boxes 1, 2, and 3). Although some non-binding instruments of international significance exist, there is no single global, enforceable regulatory framework that shapes IDG discourse. As data move across countries and continents, available national or regional regulations are rendered less efficient, inadequate, and inconsistent. In the case of national collaborations, researchers may rely on the assumption that legal norms that apply to their institutions will also hold for those of their collaborators. However, this assumption is only partially valid and often not applicable when collaborations involve multiple countries. Even though research organizations typically provide some kind of support on the development of data management plans and advice on legal aspects for technology transfer, this type of support is insufficient as the former is focused on national legislation and directives of funding agencies, whereas the latter mainly concerns advanced stages of research. Instead, given the multiple challenges related to neuroscience data sharing, it is 
Please cite this article in press as: Eke et al., International data governance for neuroscience, Neuron (2021), https://doi.org/10.1016/ j.neuron.2021.11.017

\section{CellPress}

Box 3. Sharing data obtained from non-human primate (NHP) research

Although NHPs are generally considered a powerful animal model for addressing particular neuroscience questions, research in NHPs presents serious cultural and socio-ethical concerns for many. The close phylogenetic relationship with humans underlies the use of NHPs in research: human brain disorders such as autism (Liu et al., 2016) and genetic editing for inclusion of human genes (Shi and Su, 2019) to study genetic mechanisms can inform human neurological changes. Some experiments raise significant concerns, shaped by cultural, social, legal, and ethical differences across international boundaries. In recent years, as a result of changes in legislation largely informed by public pressure, the use of NHPs in research has been reduced in the EU (Chatfield and Morton, 2018) and the United States (Lankau et al., 2014), while NHP research continues to be a staple of neuroscience research in countries in East Asia (Okano et al., 2016; Poo et al., 2016; Zhang et al., 2014). Researchers seeking to engage in NHP research may perform experiments in a permissive locale and then transfer data to more strict jurisdictions. Therefore, NHP data generated under different legal and ethical frameworks raise concerns of how such data can responsibly be shared with partners or deposited in archives hosted in areas in which such research would not have been permitted. As Rommelfanger et al. (2018) asked, should a country accept or use data collected elsewhere in a fashion that is not considered locally ethical and legal? Does this dilemma require international consensus regarding minimum standards? These are questions that border on ethics but also must be considered when developing best practices in data sharing governance. The PRIME-DE initiative is one effort raising needed awareness of this issue in NHP imaging, where funds from some agencies cannot be used to process shared data for which animal care practices, standards, and regulations are not sufficiently known or in compliance with policies (PRIMatE Data Exchange, 2020).

necessary to create mechanisms that inform and support researchers throughout all stages of the research process. Below we present a general and not exhaustive description of the different governance instruments that may apply to researchers in neuroscience. Descriptions are categorized depending on the type of governance instruments and the breadth of their scope.

International soft laws are quasi-legal instruments that are not legally binding but are encouraged as a matter of principle. Applicable soft laws include general instruments such as the UDHR (United Nations, 2021) or the Declaration of Helsinki (World Medical Association, 2021). Other examples of soft laws that are more specific to neuroscience include the Organisation for Economic Co-operation and Development (OECD) recommendations on health data governance (Organisation for Economic Co-operation and Development, 2017b) and responsible innovation in neurotechnology (Organisation for Economic Co-operation and Development, 2019). These declarations are the result of significant international consensus processes and, although not legally binding, have strong influence in the practice of organizations across the world. For instance, the European Human Brain Project (HBP) opinion on data protection (Salles et al., 2017) makes reference to the National Commission for the Protection of Human Subjects, which published "The Belmont Report: Ethical Principles and Guidelines for the Protection of Human Subjects of Research" in 1979, and the UNESCO Principle of Respect for Human Vulnerability and Personal Integrity of 2013.

National legislation on intellectual property (IP), privacy, security, and trade also defines the applicable framework for data sharing. International collaborations are particularly sensitive to discrepancies among legal frameworks in different countries. An illustrative example of this case is the EU's General Data Protection Regulation (GDPR). Although research occupies a privileged position within the GDPR, it nevertheless highlights the discrepancies in the regulations for data sharing with respect to countries outside the EU, which are often in conflict with principles governing open science (Bovenberg et al., 2020; Townend, 2018). For instance, legitimate personal data transfers from the EU to the United States were based on a legal agreement called the Privacy Shield. On July 16, 2020, the Court of Justice of the EU issued a landmark decision in the Schrems II case that invalidated the Privacy Shield decision (Fantin, 2020). The Privacy Shield was part of a list of possible legal justifications outlined in articles 44-49 of the GDPR for the transfer of personal data from the EU to other countries. This list of justifications for cross-border transfer include adequacy decisions, bilateral or multilateral agreements, specific situations on the basis of consent, public interest, vital interest of the data subject and legitimate interests pursued by the controller not overridden by the interests or rights of the data subject. In the absence of the Privacy Shield or any other form of adequacy decision, data transfers from the EU to the United States can now happen only on the basis of the other justifications on the GDPR list (Hallinan et al., 2020) and with appropriate safeguards that are mostly regarded as complex processes.

Other examples of relevant national legislations that have considerable influence on how data are shared include the US Health Insurance Portability and Accountability Act (HIPAA), which sets standards for the protection of sensitive patient health information in the United States. As illustrated in Box 2, data repositories hosted at American institutions, such as the Open Data Commons for Spinal Cord Injury (ODC-SCl; odcsci.org), require that all deposited data be compliant with HIPAA regulations. However, as shown in Figure S1, there are multiple levels and meanings of de-identification and pseudonymization. How do researchers outside the United States who have collected data under local regulations know that their data can be legally submitted to an archive such as ODC-SCI? In this category we also find clinical regulation where different entities may apply. Such legislation relies on disparate understandings of pertinent issues such as IP and core issues such as the length of time privacy must be protected. HIPAA, for example, extends privacy protection for 50 years after someone dies, whereas under the GDPR, privacy protections stop at the death of the individual. 
Please cite this article in press as: Eke et al., International data governance for neuroscience, Neuron (2021), https://doi.org/10.1016/

\begin{tabular}{|c|c|}
\hline Challenge & Description \\
\hline Ethics & $\begin{array}{l}\text { Understanding the ethical imperative for openness on one hand and the need for data protection } \\
\text { compliance on the other; also differences in organizational and cultural values as well as the ethical } \\
\text { frameworks and principles underlying the concept of data governance (Fothergill et al., 2019; Salles } \\
\text { and Farisco, 2020; Stahl et al., 2018). For example, linking among neural data, cognitive processes, } \\
\text { mental states, and mental integrity might have potential benefits but also threats, such as } \\
\text { manipulation (Yuste et al., 2017) (see also the NeuroRights Foundation at Columbia University } \\
\text { https://neurorightsfoundation.org). }\end{array}$ \\
\hline Regulations and policies & $\begin{array}{l}\text { Differences in regulations and policies, including those governing human and animal protections, } \\
\text { and different interpretations of regulations and policies. Lack of clarity on regulations and policies } \\
\text { overall and lack of notification of changes to regulations and policies (Rosenbaum, 2010). }\end{array}$ \\
\hline Different definitions of core concepts & $\begin{array}{l}\text { Core data concepts such as de-identification, anonymization, and pseudonymization may not } \\
\text { mean the same thing in different countries because of varied understanding of personal data } \\
\text { (Wiener et al., 2016). Most often "anonymization" and "de-identification" are used synonymously in } \\
\text { the literature. However, anonymization is an irreversible process, whereas de-identification makes } \\
\text { room for re-identification, which is closer in meaning to pseudonymization than anonymization } \\
\text { (Kissner, 2019; Wiener et al., 2016). Figure S1 demonstrates how these are conceptualized and } \\
\text { regulated by data protection regulations, especially by the GDPR. }\end{array}$ \\
\hline Language & $\begin{array}{l}\text { The lack of IDG can create challenges due to differences in language and interpretation among } \\
\text { partners. For example, relevant ethical and legal documents that influence data governance are in } \\
\text { different languages that individual researchers may not understand (English, German, French, } \\
\text { Japanese, Chinese, Indian, Spanish, Swedish). This highlights one of the problems posed by the } \\
\text { increasing internationalization of neuroscience research. }\end{array}$ \\
\hline Cultural diversity & $\begin{array}{l}\text { In addition to language differences, there are different regional and organizational cultural } \\
\text { differences that can affect data sharing. These differences may include social and cultural } \\
\text { constructs about the brain and mind, diversity in ethical frameworks and principles, political and } \\
\text { regional priorities, as well as approaches to intellectual property management. Sensitivity to these } \\
\text { cultural differences is needed for an effective data sharing ecosystem. }\end{array}$ \\
\hline Size, complexity, and diversity of data & $\begin{array}{l}\text { Neuroscience datasets are big and comprise large amounts of data. In addition to the technical } \\
\text { challenges of hosting and harmonizing all of these data, the size and complexity of neuroscience } \\
\text { data will likely move the scientific community toward hosting data in accessible environments such } \\
\text { as the cloud and bringing computers to the data. There are costs associated with building and } \\
\text { sustaining these infrastructures that may be beyond the reach of researchers in many geographic } \\
\text { areas. Should governments develop their own national infrastructures to support big data research } \\
\text { or let data be collected outside of government-run infrastructures? If infrastructures are funded by } \\
\text { one country, to what extent are they expected to support or subsidize global access to the data } \\
\text { hosted by them? }\end{array}$ \\
\hline
\end{tabular}

National legislation regarding data exchange can be strongly influenced by international relations. In particular, security concerns have been raised as motivation to restrict data sharing across countries. International scientific collaborations similarly may be affected because of fraying political relationships between countries or changes in data protection regulations (see Box 1).

\section{General and specific sponsor-driven policies on data sharing and governance}

Research data sharing is also shaped by policies that come from funders. More and more funders are issuing requirements for data sharing that affect all grantees. In other cases, certain funding programs may come with specific requirements for data sharing and governance. For instance, the US National Institutes of Health (NIH) BRAIN Initiative has issued a specific requirement for data sharing for recipients of funding (National Institutes of Health, 2019), even specifying into which repositories data must be deposited. The NIMH has a data archive (the NDA; National Institute of Mental Health, 2020). The NDA has specific policies with challenging requirements for data sharing mandating federal- wide assurance numbers for access. Additionally, the $\mathrm{NIH}$ recently published new data management and sharing policies (National Institutes of Health, 2020). In the HBP, these requirements include the European Commission strategy for data (European Commission, 2020) and guidelines on FAIR data management in Horizon 2020 (European Commission, DirectorateGeneral for Research \& Innovation, 2016). Table S2 shows some of the current policies and requirements for data sharing across programs in different national and geographic regions. The table focuses especially on the IBI partner programs and initiatives.

\section{Institutional policies and contracts}

Researchers wishing to share data across international borders may also encounter policies established by their home institutions that affect aspects of data governance. In some cases, institutions may have agreements in place with foreign institutions that can facilitate international collaborations. Similarly, when such agreements are not in place, researchers may have to expend considerable effort to try to negotiate memoranda of understanding (MOUs) and non-disclosure agreements (NDAs) among institutions. 
A

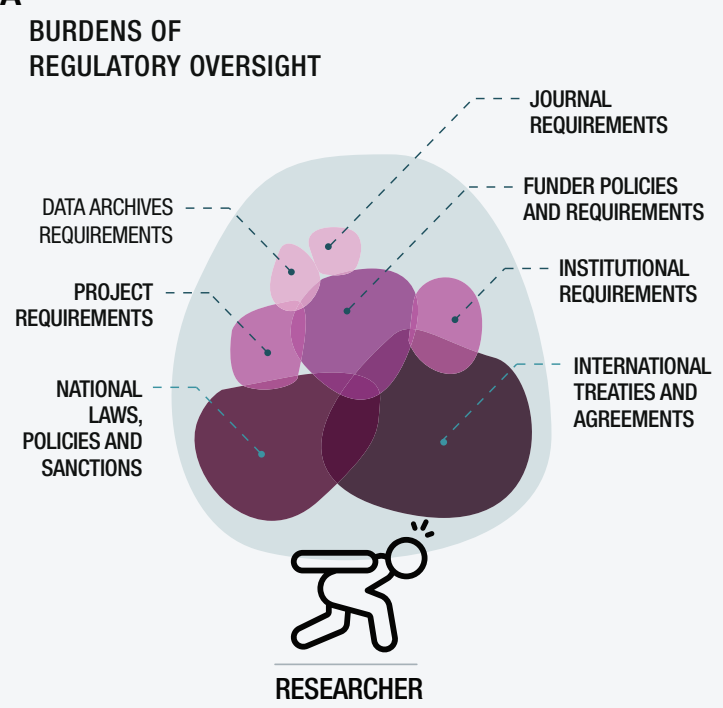

B

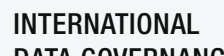

DATA GOVERNANCE

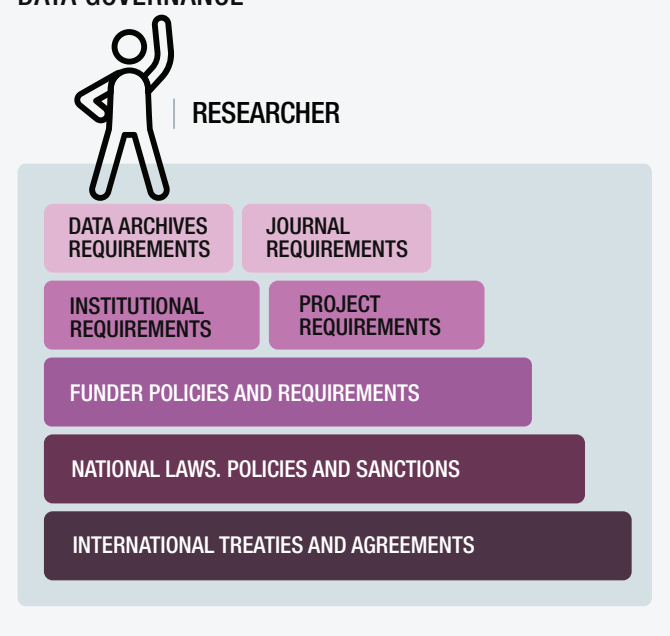

Figure 3. The cascade of regulations, policies, and norms facing researchers engaged in international data sharing

(A) Currently a researcher wishing to share data across borders is burdened by multiple layers of regulatory oversight, some of which may be competing with one another.

(B) With clear practical guidance and tools on international data governance, the researcher can instead stand on a firm foundation of ethical and legal guidelines.

\section{Project-specific policies}

Individual projects or consortia may be governed by data management and sharing rules they establish through agreement among consortium members. For example, a consortium may agree to a specific license under which data must be shared. Often consortia work through member committees to establish these agreements.

The above discussion illustrates the intricacies of the current state of brain data governance. Taking all these policies together, they represent a complex regulatory burden on the various stakeholders that must comply with them. Given the increasingly international nature of neuroscience research, researchers need training and resources that enable them to gain better understanding of the value and process of international data sharing and to navigate the various regulatory and ethical requirements associated with such activity (Figure 3).

\section{WHAT DOES AN IDG FRAMEWORK FOR NEUROSCIENCE LOOK LIKE?}

An IDG framework for neuroscience may best be thought of as a function that provides a responsible and holistic approach for all stages of the data life cycle, including collection, processing, curation, archiving and preservation, application and utilization, sharing, and deletion (Table 2). IDG should facilitate neuroscience data sharing for scientific discovery and technology transfer by reducing the burden for individual researchers or institutions. A nimble IDG framework can allow sharing while respecting the rights of subjects and cultural and ethical values. IDG will need to shape aspects of governance related to data quality (which includes its accuracy, completeness, relevancy, validity, timeliness, and consistency), secu- rity, integrity, usability, attribution, accessibility, and ultimately its trustworthiness.

From a technical perspective, IDG for neuroscience should clarify and simplify the ethical, cultural, and legal issues across the different stages of the data life cycle (see Table 2) and propose a simple workflow for addressing issues and implementing research. Whereas some issues are specific to one or two workflow stages, many of them are intricately linked. For instance, how data were collected or processed can affect the way they are shared. Sharing initiatives concerning data collected from human subjects are influenced by informed consent (Spence et al., 2018; White et al., 2020). This can also be affected by processing activities such as anonymization, de-identification, and pseudonymization. Furthermore, sharing of both human and non-human subjects can be affected by curation (standardization, identification of data controllers) and archiving (e.g., data security). Furthermore, there is a growing body of research on whether anonymization, pseudonymization, or de-identification can work or not for neuroimaging data (Eke et al., 2021; Song et al., 2015; White et al., 2020). IDG could help clarify the degree to which issues such as the potential risk for re-identification should be considered or whether they are normal within the larger research goals of the good of the society. A critical issue in the sharing stage (Table 2) is licensing of data for commercial purposes. This is implemented using different approaches across the current data repositories (Jwa and Poldrack, 2021). Underlining these commercial licensing policies are legal and ethical issues that are also different across jurisdictions. Critically, an IDG framework would clarify and communicate to the scientific community legal responsibilities and liabilities associated with sharing data across borders that 
Please cite this article in press as: Eke et al., International data governance for neuroscience, Neuron (2021), https://doi.org/10.1016/ j.neuron.2021.11.017

Neuron

\begin{tabular}{|c|c|}
\hline Data Life Cycle Stages & Neuroscience Data Governance Needs That Affect International Sharing \\
\hline Collection & $\begin{array}{l}\text { informed consent: how to collect consent for international sharing and use of data } \\
\text { sampling bias: how to represent a very heterogeneous population across ethnic groups and cultures } \\
\text { regulatory differences and legal basis for data collection: how to understand } \\
\text { the different laws for animal welfare or data protection }\end{array}$ \\
\hline Processing & $\begin{array}{l}\text { anonymization, de-identification, and pseudonymization: how to ensure that } \\
\text { subjects' privacy can be maintained while retaining scientific utility of the data } \\
\text { regulatory differences and legal basis for data processing: } \\
\text { how to understand data protection laws in different countries }\end{array}$ \\
\hline Curation & $\begin{array}{l}\text { standardization: how to understand the different standards for metadata schemes across countries } \\
\text { data curation transfer agreements: how to establish agreements } \\
\text { that allow data curation outside of the owner's nation when needed } \\
\text { security: how to ensure that risks for data breaches are minimized }\end{array}$ \\
\hline $\begin{array}{l}\text { Archiving and } \\
\text { preservation }\end{array}$ & $\begin{array}{l}\text { 1. retention policy: how long will the data be preserved? } \\
\text { 2. data controllership, stewardship, or custodianship: who owns rights on or controls data? } \\
\text { 3. funding: who is paying for data archiving, and should access be free for all users? } \\
\text { 4. security: how to ensure that risks for data leaking are minimized over time as technology changes }\end{array}$ \\
\hline Application and use & $\begin{array}{l}\text { incidental findings: how to communicate findings that pertain to the health of study participants } \\
\text { minimization: how to ensure that studies use the minimal amount of data so as } \\
\text { to minimize risks to participants (e.g., re-identification, privacy break-ins) } \\
\text { misuse: how to ensure that data are not misused or misapplied } \\
\text { for ethically, legally, or socially unacceptable purposes } \\
\text { biases in analysis and results interpretation: how to mitigate } \\
\text { data analysis bias concerns or misinterpretation of results } \\
\text { dual use: how data can be used responsibly for both civil and military application } \\
\text { commercial exploitation: what restrictions are available regarding using data for economic gain? }\end{array}$ \\
\hline Sharing & $\begin{array}{l}\text { access control: how to manage access to data, authorization, and data use } \\
\text { agreements (DUAs) across investigators, institutions, and countries } \\
\text { third-party and international sharing: how to overcome regulatory limitations to } \\
\text { sharing data to ensure effective scientific impact in international projects } \\
\text { risks for re-identification: how to prevent potential risks for re-identification } \\
\text { given advancements in machine learning and Al } \\
\text { licensing: how to approach data licensing and intellectual property concerns when required } \\
\text { attribution: how to cite and keep track of contribution } \\
\text { to data collection, processing, or curation }\end{array}$ \\
\hline Deletion & $\begin{array}{l}\text { inappropriate retention: how to ensure that data are } \\
\text { retained and deleted responsibly after they have been used }\end{array}$ \\
\hline & loss of data and unintended deletion: how to ensure resilienc \\
\hline
\end{tabular}

Several issues are at stake when data must be considered from an international perspective. Some of these issues are general to any project involving human and animal data. Yet some specific needs and challenges must be considered when crossing international borders. This table lists some of the most critical aspects that must be considered when embarking on international projects for brain research.

affect researchers and repository owners. As of today, none of this has explicitly been worked out by the neuroscience community, and as a result risks fall on individual researchers and institutions (see Boxes 1 and 2).

\section{CONCLUSION AND CRITICAL CONSIDERATIONS}

As technological advancements in $\mathrm{Al}$ and machine learning continue to expand the nature, scope, and utility of neuroscience data, we propose that the development and implementation of IDG for global neuroscience research be guided by the following considerations.

\section{Make IDG a priority}

IDG should not be an afterthought. Data governance should be addressed before (planning stages), during (project execution stage), and after (dissemination and exploitation stages) a project. When data governance is an afterthought, critical issues in the different stages of the data life cycle are missed. Again, all stakeholders (researchers, project coordinators, institutions, professional societies, publishers, infrastructure providers, funders, and other stakeholders) have a responsibility in ensuring that governance mechanisms are considered proactively. Governance mechanisms can be described in grant proposals or at the beginning of a collaboration. It is particularly important 


\section{$\infty$ CellPress} OPEN ACCESS
Neuron Review that a defined data governance framework that aligns with accepted IDG be established at the beginning of any project involving international collaborators or with the potential to share data internationally. Infrastructure and institutional policies can also play an instrumental role in ensuring that IDG is not an afterthought. Although any changes may require the identification of funding and resources that are required to develop informed IDG plans, concrete next steps to addressing policy changes and raising awareness among appropriate stakeholders are included below.

\section{Suggestions for practical actions}

- Expand the funding initiatives to support the development and implementation of IDG tools and services for neuroscience. Programs at the scale of international coordination as well as multidisciplinary work would be ideal. Efforts to develop and implement such tools would require leadership and support for researchers, as well as the involvement of legal, security, economic, ethics, and technology experts across national borders.

- Integrating IDG into research project planning, similar to how data management plans are required in grant applications, would facilitate responsible IDG within research.

- Establish offices of data governance at institutions, data repositories, neurotechnology companies, research projects, funders, and other relevant bodies.

\section{Develop principles for IDG}

There is a need for simple and clear international governance principles to maximize openness and access to data for the good of scientific progress. These principles should cover ethical, scientific, technical, legal, and sharing requirements. The development of IDG principles should be developed by identifying and involving the multiple stakeholders involved (e.g., researchers, civil society groups, funders, journals, institutional representatives, technologists). Given the differences in culture and legal systems across nations, and given the many stakeholders involved in processing neuroscience data (researchers, institutions, infrastructure providers, funders, and local governments), IDG for neuroscience would need to be defined by involving multiple communities and representatives from various nations and cultures (Fothergill et al., 2019; Stahl et al., 2019). There is a need to acknowledge and be responsive to the many different needs and socio-cultural and political dynamics that shape the diverse ethical principles and laws associated with data. Therefore, inclusive dialogues with different stakeholders from different cultures and disciplinary backgrounds should characterize the development of IDG principles for neuroscience.

\section{Suggestion for practical action}

- Initiate multi-stakeholder partnerships and collaborations to develop legal, ethical, technical, organizational, and cultural principles that can shape IDG for neuroscience data. Primary stakeholders include transnational neuroscience researchers, organizations (e.g., the OECD, UNE SCO), technical societies (IEEE, INCF), archives, projects and platforms involved in data standards, sharing and analysis (OpenNeuro.org, brainlife.io, conp.ca, ebrains. eu, PRIMatE Data Exchange [PRIME-DE]), and scientific organizations associated with brain research.

\section{Develop practical tools and guidance for} streamlined IDG

Developing simple, easy-to-use tools that allow researchers or other stakeholders to navigate IDG on a case-by-case basis (i.e., when sharing data between pairs of countries) is critical. These tools should help clarify the different and competing ethical and legal principles involved in cross-border transfers for easier implementation. Researchers and funders alike need practical answers to complex recurrent issues (e.g., consent, anonymization, access control, human rights, security). Examples of such would be quick guides on how to share data between pairs of countries, what to share or receive, how to establish consent forms that would allow open sharing of data (see the Open Brain Consent initiative; Bannier et al., 2021). These tools will ensure that relevant information is available to the scientific community when they are planning an international project. Ideally, this effort would include the development of semi-automated methods for the analysis of regulatory documents and ethical perspectives across countries, and it would involve input and guidance from experts in navigating international data sharing issues. Individuals with such expertise exist, but they are generally scattered and may be hard to find. Fostering a network of these individuals and integrating them into neuroscience meetings can help provide guidance to those trying to work across borders.

\section{Suggestions for practical actions}

- Create a global alliance (a consortium) that will guide the development of technical standards and establish data governance best practices. The new consortium should be organized at a grass-roots level, led by scientists, and endorsed by neuroscience organizations. The consortium would also need to involve funders and policy makers to represent the wide range of interests in neuroscience data. The consortium would be synergistic but not overlapping with the issues already addressed by the GA4GH (GA4GH.org).

- Develop a sustainable federated data catalog of neuroscience data from brain initiatives and other resources around the world, establish governance to maintain metadata standards for FAIR data sharing, and develop and disseminate tutorials, training materials, and educational activities for dataset publishing and data reuse.

- Develop a neuroscience technical IDG toolkit. Among other things, the toolkit would guide researchers on IDG across the data life cycle (Table 2).

- Develop a regulatory and ethical IDG toolkit. This would be defined by a series of documents that would serve researchers to navigate issues related to IDG.

\section{Increase awareness and education on IDG}

There is a need to promote a cultural shift in the scientific community so as to increase awareness of the importance of IDG, 


\section{Neuron} Review

given the increasingly international nature of neuroscience research. Issues of cultural and social diversity across nations are especially important, including considerations of race, age, and gender when defining policies and planning research projects. Such changes in awareness and attitude will require establishing educational mechanisms for responsible open neuroscience data sharing (Choudhury et al., 2014). Developing a set of educational resources is necessary not only to engender cultural change but also to educate investigators on how IDG works and provide key examples using specific research data samples and types. These educational resources should explain both the value of sharing data and the ethical and legal responsibilities of the parties involved. Furthermore, the educational resources could promote the use of data for educational purposes as well as research. This process of data upcycling (Avesani et al., 2019) is critical to training a new generation of scientists with a global mind-set on the globalized nature of the research enterprise and creating a culture that attracts and retains a diversity of thinking, heritage, and skill sets in the neuroscience community. Furthermore, this will effectively accelerate scientific discovery by attracting a multitude of opinions and by bringing higher education closer to the most cutting-edge research data.

\section{Suggestions for practical actions}

- Integrate IDG into educational and professional training activities organized by professional societies and educational institutions (e.g., neuroscience curricula for graduate and undergraduate programs, professional development for postdoctoral scholars), including formal IDG certification educational training courses.

- Encourage neuroscience conference organizers, funders, and other initiatives to consider and support targeted training programs dedicated to IDG.

- Offer efficient, accessible, up-to-date data governance training and associated materials for neuroscientists, especially those working in countries with less economic capacity, through international scientific organizations and societies.

The establishment of an IDG framework guided by the above considerations will require dedicated efforts and significant resources. Fortunately, neuroscience has established internationally focused organizations such as the $I B I$, the INCF, and societies such as the International Brain Research Organization (IBRO), which can facilitate discussions and work toward the development of IDG. The IBI serves as a coordinator across large-scale neuroscience initiatives, with the aim of creating impact that broadly benefits neuroscience. The above recommendations will be pursued by participants in the $\mathrm{IBI}$ network, especially in the early stages, but most points outlined here will require a broader community effort. The recommendations would be then developed and implemented in partnership with neuroscience-specific organizations (e.g., scientific societies such as the Society for Neuroscience, IBRO, coordinating bodies such as the INCF), data standards and sharing projects (e.g., OpenNeuro.org, brainlife.io, conp.ca, ebrains.eu, NeuroMorpho.org, PRIME$\mathrm{DE})$, the private sector and professional organizations (e.g., in- dustry, the IEEE), data policy experts, and transnational bodies (e.g., the OECD, UNESCO).

\section{SUPPLEMENTAL INFORMATION}

Supplemental information can be found online at https://doi.org/10.1016/j. neuron.2021.11.017.

\section{ACKNOWLEDGMENTS}

The authors' views are personal views and do not necessarily represent those of the organizations with which they are affiliated, such as the National Science Foundation (NSF), National Institutes of Health (NIH) or US federal government. We thank Kenji Doya, Megumi Maruyama, and Nargis Akter for administrative support. A.M. and A.B. are supported by the Kavli Foundation. D.O.E. and J.G.B. are supported by the European Union's Horizon 2020 Framework Programme for Research and Innovation under Specific Grant Agreement (SGA) 945539 (HBP, SGA3). O.R. is supported by the National Institute of Neurological Disorders and Stroke of the NIH under award U24NS120057. A.J.H. is supported by a Principal Research Fellowship and Ideas Grant from the National Health and Medical Research Council (NHMRC) and by the DHB Foundation (Equity Trustees) and Flicker of Hope Foundation. S.L.H. is supported by Krembil Foundation funding. T.H. is supported by the Strategic International Brain Science Research Promotion Program (21dm0307003h0004 and 21dm0307004h0003) and Brain Mapping by Integrated Neurotechnologies for Disease Studies (21dm0207070h0001) from the Japan Agency for Medical Research and Development. F.P. is supported by NSF grants IIS 1636893, IIS 1912270, and BCS 1734853, NIH National Institute of Biomedical Imaging and Bioengineering (NIBIB) grant 1R01EB029272, NIH NIMH 1R01MH126699, and a Microsoft Investigator Fellowship.

\section{DECLARATION OF INTERESTS}

M.E.M. is a founder and has equity interest in SciCrunch.com, a technology startup providing services in support of Research Resource Identifiers and reproducible science.

\section{REFERENCES}

Abrams, M.B., Bjaalie, J.G., Das, S., Egan, G.F., Ghosh, S.S., Goscinski, W.J., Grethe, J.S., Kotaleski, J.H., Ho, E.T.W., Kennedy, D.N., et al. (2021). A standards organization for open and FAIR neuroscience: the International Neuroinformatics Coordinating Facility. Neuroinformatics. Published online January 27, 2021. https://doi.org/10.1007/s12021-020-09509-0.

All European Academies; European Academies Science Advisory Council; Federation of European Academies of Medicine (2021). International sharing of personal health data for research. https://easac.eu/fileadmin/PDF_s/ reports_statements/Health_Data/International_Health_Data_Transfer_2021_ web.pdf.

Amunts, K., Knoll, A.C., Lippert, T., Pennartz, C.M.A., Ryvlin, P., Destexhe, A. Jirsa, V.K., D'Angelo, E., and Bjaalie, J.G. (2019). The Human Brain Projectsynergy between neuroscience, computing, informatics, and brain-inspired technologies. PLoS Biol. 17, e3000344.

Ascoli, G.A., Maraver, P., Nanda, S., Polavaram, S., and Armañanzas, R (2017). Win-win data sharing in neuroscience. Nat. Methods 14, 112-116.

Avesani, P., McPherson, B., Hayashi, S., Caiafa, C.F., Henschel, R., Garyfallidis, E., Kitchell, L., Bullock, D., Patterson, A., Olivetti, E., et al. (2019). The open diffusion data derivatives, brain data upcycling via integrated publishing of derivatives and reproducible open cloud services. Sci. Data 6, 69.

Bannier, E., Barker, G., Borghesani, V., Broeckx, N., Clement, P., Emblem, K.E., Ghosh, S., Glerean, E., Gorgolewski, K.J., Havu, M., et al. (2021). The Open Brain Consent: informing research participants and obtaining consent to share brain imaging data. Hum. Brain Mapp. 42, 1945-1951.

Bovenberg, J., Peloquin, D., Bierer, B., Barnes, M., and Knoppers, B.M. (2020). How to fix the GDPR's frustration of global biomedical research. Science $370,40-42$ 
Chatfield, K., and Morton, D. (2018). The Use of Non-human Primates in Research. In Ethics Dumping: Case Studies from North-South Research Collaborations, D. Schroeder, J. Cook, F. Hirsch, S. Fenet, and V. Muthuswamy, eds. (Springer), pp. 81-90.

Choudhury, S., Fishman, J.R., McGowan, M.L., and Juengst, E.T. (2014). Big data, open science and the brain: lessons learned from genomics. Front. Hum. Neurosci. 8, 239. https://doi.org/10.3389/fnhum.2014.00239.

Eglen, S.J., Marwick, B., Halchenko, Y.O., Hanke, M., Sufi, S., Gleeson, P., Silver, R.A., Davison, A.P., Lanyon, L., Abrams, M., et al. (2017). Toward standard practices for sharing computer code and programs in neuroscience. Nat. Neurosci. $20,770-773$.

Eiss, R. (2020). Confusion over Europe's data-protection law is stalling scientific progress. Nature 584, 498 .

Eke, D., Aasebø, I.E.J., Akintoye, S., Knight, W., Karakasidis, A., Mikulan, E., Ochang, P., Ogoh, G., Oostenveld, R., Pigorini, A., et al. (2021). Pseudonymisation of neuroimages and data protection: increasing access to data while retaining scientific utility. Neuroimage Rep. 1, 100053.

European Commission (2020). Communication from the Commission to the European Parliament, the Council, the European Economic and Social Committee and the Committee of the Regions: a European strategy for data. https://eur-lex.europa.eu/legal-content/EN/TXT/PDF/?uri=CELEX:52020DC0 066\&from $=E N$.

European Commission, Directorate-General for Research \& Innovation (2016). Guidelines on FAIR data management in Horizon 2020. https://ec.europa.eu/ research/participants/data/ref/h2020/grants_manual/hi/oa_pilot/h2020-hi-oadata-mgt_en.pdf.

Fantin, S. (2020). Data Protection Commissioner v Facebook Ireland Limited, Maximillian Schrems: AG discusses the validity of standard contractual clauses and raises concerns over privacy shield (C-311/18 Schrems II, opinion of AG Saugmandsgaard Øe). Eur. Data Prot. Law Rev. 6, 325-331.

Ferguson, A.R., Nielson, J.L., Cragin, M.H., Bandrowski, A.E., and Martone, M.E. (2014). Big data from small data: data-sharing in the 'long tail' of neuroscience. Nat. Neurosci. 17, 1442-1447.

Fothergill, B.T., Knight, W., Stahl, B.C., and Ulnicane, I. (2019). Responsible data governance of neuroscience big data. Front. Neuroinform. 13, 28.

Gorgolewski, K.J., Auer, T., Calhoun, V.D., Craddock, R.C., Das, S., Duff, E.P., Flandin, G., Ghosh, S.S., Glatard, T., Halchenko, Y.O., et al. (2016). The Brain Imaging Data Structure, a format for organizing and describing outputs of neuroimaging experiments. Sci. Data 3, 160044.

Halchenko, Y.O., and Hanke, M. (2012). Open is not enough. Let's take the next step: an integrated, community-driven computing platform for neuroscience. Front. Neuroinform. 6, 22.

Hallinan, D., Bernier, A., Cambon-Thomsen, A., Crawley, F.P., Dimitrova, D., Medeiros, C.B., Nilsonne, G., Parker, S., Pickering, B., and Rennes, S. (2020). International transfers of health research data following Schrems II: a problem in need of a solution. Eur. J. Hum. Genet. 29, 1502-1509.

Hallinan, D., Akintoye, S., Stahl, B.C., and Eke, D. (2021). Neuroexceptionalism: framing an emergent debate. https://papers.ssrn.com/sol3/papers. cfm?abstract_id $=3909816$.

Haynes, J.-D., Sakai, K., Rees, G., Gilbert, S., Frith, C., and Passingham, R.E. (2007). Reading hidden intentions in the human brain. Curr. Biol. 17, 323-328.

lenca, M., and Andorno, R. (2017). Towards new human rights in the age of neuroscience and neurotechnology. Life Sci. Soc. Policy 13, 5 .

lenca, M., Vayena, E., and Blasimme, A. (2018). Big data and dementia: charting the route ahead for research, ethics, and policy. Front. Med. (Lausanne) $5,13$.

Institute of Electrical and Electronics Engineers Standards Association (2020). Standards roadmap: neurotechnologies for brain-machine interfacing. IEEE SA Industry Connections Activity No. IC17-007. https://standards.ieee.org/ content/dam/ieee-standards/standards/web/documents/presentations/ieeeneurotech-for-bmi-standards-roadmap.pdf.

International Brain Initiative (2020). International Brain Initiative: an innovative framework for coordinated global brain research efforts. Neuron 105, 212-216.
Jwa, A., and Poldrack, R. (2021). The spectrum of data sharing policies in neuroimaging data repositories. PsyArXiv. https://doi.org/10.31234/osf.io/cnuy7.

Kellmeyer, P. (2018). Big brain data: on the responsible use of brain data from clinical and consumer-directed neurotechnological devices. Neuroethics 14, 83-98.

Kietzmann, T.C., McClure, P., and Kriegeskorte, N. (2019). Deep neural networks in computational neuroscience. In Oxford Research Encyclopedia of Neuroscience (New York: Oxford University Press).

Kissner, L. (2019). Deidentification versus anonymization. https://iapp.org/ news/a/de-identification-vs-anonymization/.

Klapwijk, E.T., van den Bos, W., Tamnes, C.K., Raschle, N.M., and Mills, K.L. (2021). Opportunities for increased reproducibility and replicability of developmental neuroimaging. Dev. Cogn. Neurosci. 47, 100902.

Knoppers, B.M., and Thorogood, A.M. (2017). Ethics and big data in health. Curr. Opin. Syst. Biol. 4, 53-57.

Lankau, E.W., Turner, P.V., Mullan, R.J., and Galland, G.G. (2014). Use of nonhuman primates in research in North America. J. Am. Assoc. Lab. Anim. Sci. 53, 278-282.

Liu, Z., Li, X., Zhang, J.-T., Cai, Y.-J., Cheng, T.-L., Cheng, C., Wang, Y., Zhang, C.-C., Nie, Y.-H., Chen, Z.-F., et al. (2016). Autism-like behaviours and germline transmission in transgenic monkeys overexpressing MeCP2. Nature 530, 98-102

Mackenzie, A. (2019). The data-sharing problem in neuroscience (New York: Simons Foundation). https://www.simonsfoundation.org/2019/10/04/the-datasharing-problem-in-neuroscience/.

Marblestone, A.H., Wayne, G., and Kording, K.P. (2016). Toward an integration of deep learning and neuroscience. Front. Comput. Neurosci. 10, 94.

McClure, S.M., Li, J., Tomlin, D., Cypert, K.S., Montague, L.M., and Montague, P.R. (2004). Neural correlates of behavioral preference for culturally familiar drinks. Neuron 44, 379-387.

McDougal, R.A., Bulanova, A.S., and Lytton, W.W. (2016). Reproducibility in computational neuroscience models and simulations. IEEE Trans. Biomed. Eng. 63, 2021-2035.

Milham, M.P., Craddock, R.C., Son, J.J., Fleischmann, M., Clucas, J., Xu, H., Koo, B., Krishnakumar, A., Biswal, B.B., Castellanos, F.X., et al. (2018). Assessment of the impact of shared brain imaging data on the scientific literature. Nat. Commun. 9, 2818.

Mittelstadt, B.D., and Floridi, L. (2016). The ethics of big data: current and foreseeable issues in biomedical contexts. In The Ethics of Biomedical Big Data, B.D. Mittelstadt and L. Floridi, eds. (Cham, Switzerland: Springer International), pp. 445-480.

National Institute of Mental Health (2020). NIMH Data Archive: data sharing terms and conditions. https://s3.amazonaws.com/nda.nih.gov/Documents/ $\mathrm{NDA}+$ Data+Sharing+Terms+and+Conditions+01.01.20.pdf.

National Institutes of Health (2019). Notice of data sharing policy for the BRAIN Initiative. https://grants.nih.gov/grants/guide/notice-files/NOT-MH-19-010.html.

National Institutes of Health (2020). Final NIH policy for data management and sharing. https://grants.nih.gov/grants/guide/notice-files/NOT-OD-21-013.html.

Nichols, T.E., Das, S., Eickhoff, S.B., Evans, A.C., Glatard, T., Hanke, M., Kriegeskorte, N., Milham, M.P., Poldrack, R.A., Poline, J.-B., et al. (2017). Best practices in data analysis and sharing in neuroimaging using MRI. Nat. Neurosci. 20, 299-303.

Okano, H., Sasaki, E., Yamamori, T., Iriki, A., Shimogori, T., Yamaguchi, Y., Kasai, K., and Miyawaki, A. (2016). Brain/MINDS: a Japanese National Brain Project for Marmoset Neuroscience. Neuron 92, 582-590.

Organisation for Economic Co-operation and Development (2017a). Co-ordination and support of international research data networks. OECD Science, Technology and Innovation Policy Papers No. 51. https://www.oecd-ilibrary org/docserver/e92fa89e-en. pdf?expires=1637436659\&id=id\&accname=quest $\&$ checksum=BAB005A22E1F8DD52C031A3035B808B8. 


\section{Neuron}

Organisation for Economic Co-operation and Development (2017b). OECD recommendation on health data governance. https://www.oecd.org/els/ health-systems/health-data-governance.htm.

Organisation for Economic Co-operation and Development (2019). OECD recommendation on responsible innovation in neurotechnology. https://www.oecd.org/science/recommendation-on-responsible-innovation-inneurotechnology.htm.

Paninski, L., and Cunningham, J.P. (2018). Neural data science: accelerating the experiment-analysis-theory cycle in large-scale neuroscience. Curr. Opin. Neurobiol. 50, 232-241.

Poldrack, R.A., and Gorgolewski, K.J. (2014). Making big data open: data sharing in neuroimaging. Nat. Neurosci. 17, 1510-1517.

Poline, J.-B., Breeze, J.L., Ghosh, S., Gorgolewski, K., Halchenko, Y.O., Hanke, M., Haselgrove, C., Helmer, K.G., Keator, D.B., Marcus, D.S., et al. (2012). Data sharing in neuroimaging research. Front. Neuroinform. 6, 9.

Poo, M.-M., Du, J.-L., Ip, N.Y., Xiong, Z.-Q., Xu, B., and Tan, T. (2016). China Brain Project: basic neuroscience, brain diseases, and brain-inspired computing. Neuron 92, 591-596.

PRIMatE Data Exchange (PRIME-DE) Global Collaboration Workshop and Consortium (2020). Accelerating the evolution of nonhuman primate neuroimaging. Neuron 105, 600-603.

Rabesandratana, T. (2019). European data law is impeding studies on diabetes and Alzheimer's, researchers warn. Science https://www.science.org/ content/article/european-data-law-impeding-studies-diabetes-and-alzheimers-researchers-warn.

Rommelfanger, K.S., Jeong, S.J., Ema, A., Fukushi, T., Kasai, K., Ramos, K.M., Salles, A., and Singh, I.; Global Neuroethics Summit Delegates (2018). Neuroethics questions to guide ethical research in the international brain initiatives. Neuron 100, 19-36.

Rosenbaum, S. (2010). Data governance and stewardship: designing data stewardship entities and advancing data access. Health Serv. Res. 45, 1442-1455.

Salerno, J., Knoppers, B.M., Lee, L.M., Hlaing, W.M., and Goodman, K.W. (2017). Ethics, big data and computing in epidemiology and public health. Ann. Epidemiol. 27, 297-301.

Salles, A., and Farisco, M. (2020). Of ethical frameworks and neuroethics in big neuroscience projects: a view from the HBP. AJOB Neurosci. 11, 167-175.

Salles, A., Stahl, B., Bjaalie, J., Domingo-Ferrer, J., Rose, N., Rainey, S., and Spranger, T. (2017). Opinion and action plan on 'data protection and privacy' (Human Brain Project). https://sos-ch-dk-2.exo.io/public-website-production/ filer public/24/0e/240e2eaa-8a10-4a17-87bc-b056a3f0cc8c/opinion on data protection_and_privacy_done_01.pdf.

Schreiber, D., Fonzo, G., Simmons, A.N., Dawes, C.T., Flagan, T., Fowler, J.H., and Paulus, M.P. (2013). Red brain, blue brain: evaluative processes differ in Democrats and Republicans. PLoS ONE 8, e52970.

Secretariat of the Convention on Biological Diversity (2011). Nagoya protocol on access to genetic resources and the fair and equitable sharing of benefits arising from their utilization to the Convention on Biological Diversity: text and annex. https://www.cbd.int/abs/doc/protocol/nagoya-protocol-en.pdf.

Shi, L., and Su, B. (2019). A transgenic monkey model for the study of human brain evolution. Zool. Res. 40, 236-238.
Song, X., Wang, J., Wang, A., Meng, Q., Prescott, C., Tsu, L., and Eckert, M.A. (2015). DelD - a data sharing tool for neuroimaging studies. Front. Neurosci. $9,325$.

Spence, O., Onwuchekwa Uba, R., Shin, S., and Doshi, P. (2018). Patient consent to publication and data sharing in industry and $\mathrm{NIH}$-funded clinical trials. Trials 19, 269.

Stahl, B.C., Rainey, S., Harris, E., and Fothergill, B.T. (2018). The role of ethics in data governance of large neuro-ICT projects. J. Am. Med. Inform. Assoc. 25, 1099-1107.

Stahl, B.C., Akintoye, S., Fothergill, B.T., Guerrero, M., Knight, W., and Ulnicane, I. (2019). Beyond research ethics: dialogues in Neuro-ICT research. Front. Hum. Neurosci. 13, 105.

Statt, N. (2017). Kernel is trying to hack the human brain -but neuroscience has a long way to go. The Verge. https://www.theverge.com/2017/2/22/14631122/ kernel-neuroscience-bryan-johnson-human-intelligence-ai-startup.

Teeters, J.L., Godfrey, K., Young, R., Dang, C., Friedsam, C., Wark, B., Asari, H., Peron, S., Li, N., Peyrache, A., et al. (2015). Neurodata without borders: creating a common data format for neurophysiology. Neuron 88, 629-634.

Townend, D. (2018). Conclusion: harmonisation in genomic and health data sharing for research: an impossible dream? Hum. Genet. 137, 657-664.

United Nations (2021). Universal Declaration of Human Rights. https://www. un.org/en/about-us/universal-declaration-of-human-rights.

Urban, T. (2017). Neuralink and the brain's magical future. Wait but Why. https://waitbutwhy.com/2017/04/neuralink.html.

Vayena, E., Gasser, U., Wood, A., O'Brien, D.R., and Altman, M. (2016). Elements of a new ethical framework for big data research. Wash. Lee Law Rev. 72 (3). https://scholarlycommons.law.wlu.edu/wlulr-online/vol72/iss3/5/.

Wexler, A., and Reiner, P.B. (2019). Oversight of direct-to-consumer neurotechnologies. Science 363, 234-235.

White, T., Blok, E., and Calhoun, V.D. (2020). Data sharing and privacy issues in neuroimaging research: opportunities, obstacles, challenges, and monsters under the bed. Hum. Brain Mapp. Published online July 4, 2020. https://doi. org/10.1002/hbm.25120.

Wiener, M., Sommer, F.T., Ives, Z.G., Poldrack, R.A., and Litt, B. (2016) Enabling an open data ecosystem for the neurosciences. Neuron 92, 617-621.

Wilkinson, M.D., Dumontier, M., Aalbersberg, I.J.J., Appleton, G., Axton, M., Baak, A., Blomberg, N., Boiten, J.-W., da Silva Santos, L.B., Bourne, P.E., et al. (2016). The FAIR guiding principles for scientific data management and stewardship. Sci. Data 3, 160018.

World Medical Association (2021). WMA Declaration of Helsinki-ethica principles for medical research involving human subjects. https://www.wma. net/policies-post/wma-declaration-of-helsinki-ethical-principles-for-medicalresearch-involving-human-subjects/.

Yuste, R., Goering, S., Arcas, B.A.Y., Bi, G., Carmena, J.M., Carter, A., Fins, J.J., Friesen, P., Gallant, J., Huggins, J.E., et al. (2017). Four ethical priorities for neurotechnologies and Al. Nature 551, 159-163.

Zhang, X.-L., Pang, W., Hu, X.-T., Li, J.-L., Yao, Y.-G., and Zheng, Y.-T. (2014) Experimental primates and non-human primate (NHP) models of human diseases in China: current status and progress. Dongwuxue Yanjiu 35, 447-464. 